\title{
High Resolution Flexible 3-RRR Planar Parallel Micro-Stage in Near Singular Configuration for Resolution Improvement
}

\author{
Stéphane Ronchi ${ }^{(1)(2)}$, Olivier Company ${ }^{(1)}$, Sébastien $\operatorname{Krut}^{(1)}$, François Pierrot ${ }^{(1)}$ and Alain Fournier ${ }^{(1)}$ \\ (1) : LIRMM UMR 5506 CNRS - UM2 \\ 161 rue Ada, 34392 Montpellier, France \\ \{ronchi, company, krut, pierrot, fournier\}@lirmm.fr \\ ${ }^{(2)}$ : Development Department \\ NBS Technologies SAS \\ Avenue Villevieille, 13106 Rousset, France
}

\begin{abstract}
We focus on the micro-stage of a new high resolution positioning machine. One of its key features is the use of flexible circular notch hinges for passive joints. After a reminder of the micro-stage architecture and its inverse position and velocity kinematics solutions, a force model is developed for the choice of the actuators. This model takes into account the flexible $R$ (Revolute) joints modeled as torsional springs and the external forces applied on the traveling plate. Simulations are conducted to evaluate both kinds of forces and result in the choice of the appropriate actuator. The final design of the micro-stage is presented as well as the obtained prototype.
\end{abstract}

Keywords: force modeling, flexible links, planar, parallel, near singular configuration, high resolution, positioning mechanism.

\section{INTRODUCTION AND REMINDER OF THE ARCHITECTURE}

Positioning problems are becoming crucial in many industries such as microelectronics. At the present time, research issues concern accuracy, repeatability, resolution and speed for precision positioning mechanisms.

We propose to address this important issue with a novel macro/micro machine architecture composed of two stages: a macro-stage for coarse motion presented by authors [1] and a micro-stage for fine motion. We focus in this paper on the micro-stage that will achieve the high resolution and fast positioning. New micro-stage architecture has been presented by the authors in [2], based on the combination of different features:

- Planar parallel mechanism.

- Near singular configuration.

- Flexible joints.

The choices for such a combination of features have been detailed in [2]. We will focus in this paper on the flexible joints, and more precisely on the force modeling. The actuator forces necessary to move the micro-stage mechanism will be evaluated.

Concerning flexible links, several bibliographic papers were written to model their behavior. We can cite two major books written by Howell [3] and Henein [4] that try to cover the complete range of flexible links, as well as some modeling methods for multiple instances of flexibles links in assemblies and examples on realized prototypes. Another interesting approach has been more focused on flexible links realized by machining down of material on a part: circular notch flexure hinge [5]. Criteria for the choice of the link material, the behavior of elastic beams and their use to realize flexible links are detailed. A prototype of sensor realized using flexible links is presented. Two prototypes of spatial parallel manipulators have been presented by Harai et al [6] and Chung et al [7]. The first one possesses 3-DOF (Degrees Of Freedom) in translation along the three axis of space. The second one also possesses 3-DOF, but they are $1 \mathrm{~T}-2 \mathrm{R}$ ( $\mathrm{T}$ stands for translation and $\mathrm{R}$ for rotation). Both mechanisms contain monolithic (composed of one unique part) kinematic chains made of flexible links. To get closer from what we want to work on, two studies written by Hesselbach et al [8] and Yi et al [9] concern planar parallel structures utilizing flexible links. The first one proposes a modeling of flexible notch hinge and then includes it into a planar parallel mechanism. The second one investigates a planar parallel flexible links mechanism with two different kinds of flexible links: one composed of 1-DOF (1R) flexible links, and one composed of 2-DOF (1T-1R) flexible links. The stiffness model is investigated for both kinds of flexible links. Finally, a force-torque sensor has been developed using a spatial parallel mechanism in a near singular configuration using flexure hinges by Ranganath [10]. This concept has been used to build a prototype based on the Stewart platform. Some experimental results showed the sensitivity of the structure relatively to the externally applied forces and moments.

This paper is divided in four parts. Section II will remind the geometrical modeling of one kinematic chain as well as the notations used to obtain the models that have been detailed for the inverse position and velocity kinematics solutions. In section III, we will address the flexible links force modeling, and the evaluation of forces necessary to actuate the micro-stage (externally applied forces and flexible links generated forces). Finally, section IV will present the final design for the micro-stage, its CAD (Computer Aided Design) view as well as the realized prototype. Future work will also be presented. 


\section{GEOMETRICAL MODELING AND JACOBEAN MATRIX $\boldsymbol{J}_{\text {micro }}$}

A. Presentation of the geometry

The geometry chosen to represent one kinematic chain of the micro-stage is detailed in Fig. 1. Fig. 2 represents the same kinematic chain, once manufactured and assembled.



Fig. 1 Notations used in the different modelings



Fig. 2 Flexible machined kinematic chain

- $\quad u, v, w, s, u_{\text {ortho }}$ respectively represents the unitary vectors of parts going from $\mathrm{A}$ to $\mathrm{B}, \mathrm{B}$ to $\mathrm{C}, \mathrm{G}$ to $\mathrm{F}$, $\mathrm{F}$ to $\mathrm{E}$, and $\mathrm{H}$ to $\mathrm{E}$. $\boldsymbol{w}_{\text {ortho }}$ and $\boldsymbol{s}_{\text {ortho }}$ respectively represents the unitary vectors orthogonal to $\boldsymbol{w}$ and $\boldsymbol{s}$.

- $\mathrm{G}$ is the origin of the actuator. Points $\mathrm{A}$ and $\mathrm{G}$ are attached to the frame. $\mathrm{D}$ is the center of the traveling plate (moving element).

- $\mathrm{L}_{1}, \mathrm{~L}_{2}, \mathrm{~L}_{3}, \mathrm{~L}_{4}$ and $\mathrm{L}_{\text {act }}$ are respectively the lengths of segments going from $\mathrm{A}$ to $\mathrm{B}, \mathrm{B}$ to $\mathrm{C}, \mathrm{E}$ to $\mathrm{F}, \mathrm{H}$ to $\mathrm{E}$ and $\mathrm{A}$ to $\mathrm{H}$

\section{B. Matrix $\boldsymbol{J}_{\text {micro }}$}

With the geometry defined in Fig. 1, we were able to write the inverse position and velocity kinematics solutions. To complete these models, we separated the whole micro-stage into two sub-mechanisms. One is composed of the planar parallel 3-RRR mechanism represented in Fig. 3 by the letters $\mathrm{A}_{\mathrm{i}} \mathrm{B}_{\mathrm{i}} \mathrm{C}_{\mathrm{i}} \mathrm{D}_{\mathrm{i}}(i=1,2,3$, represents the number of the studied kinematic chain), and the second one is the decoupled actuating PRR (underlined means actuated) mechanism represented in Fig. 3 by the letters $\mathrm{A}_{\mathrm{i}} \mathrm{E}_{\mathrm{i}} \mathrm{F}_{\mathrm{i}} \mathrm{G}_{\mathrm{i}}$.

The inverse velocity kinematics solutions give the actuators velocity parameter $\dot{q}_{a c t i}$ for a given set of velocity $(\dot{x}, \dot{y}, \dot{\theta})$ of the point of interest of the traveling plate $\mathrm{D}$ (also known as the operational point).

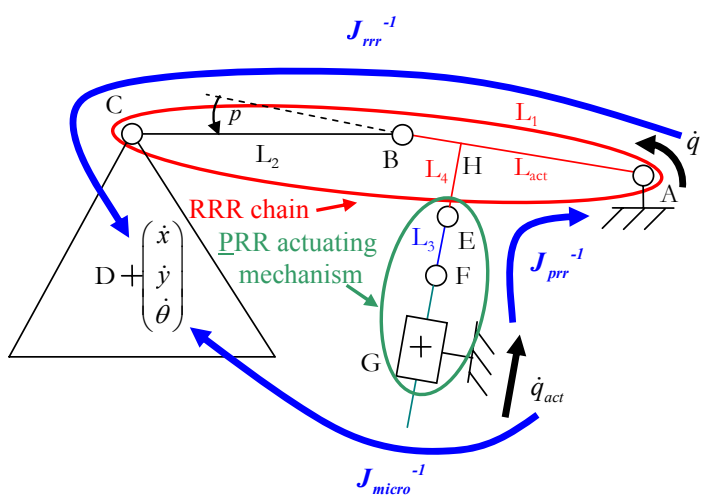

Fig. 3 Sub-mechanism and matrix decomposition

While writing the inverse velocity kinematics solutions, we managed to write the following relation (see [2] for a complete and detailed modeling):

with

$$
\begin{gathered}
\dot{\boldsymbol{q}}_{\text {act }}=\boldsymbol{J}_{\text {micro }}{ }^{-1} \dot{\boldsymbol{x}} \\
\dot{\boldsymbol{q}}_{\text {act }}=\left(\begin{array}{l}
\dot{q}_{a c t 1} \\
\dot{q}_{a c t 2} \\
\dot{q}_{a c t 3}
\end{array}\right), \dot{\boldsymbol{x}}=\left(\begin{array}{c}
\dot{x} \\
\dot{y} \\
\dot{\theta}
\end{array}\right), \\
\boldsymbol{J}_{\text {micro }}^{-1}=\boldsymbol{J}_{\text {prr }}{ }^{-1} \boldsymbol{J}_{\text {rrr }}^{-1}
\end{gathered}
$$

and

where $\boldsymbol{J}_{r r r}^{-1}=\boldsymbol{J}_{q \text { rrr }}{ }^{-1} \boldsymbol{J}_{\boldsymbol{x} r r}$ and $\boldsymbol{J}_{p r r}{ }^{-1}=\boldsymbol{J}_{\text {qact }}{ }^{-1} \boldsymbol{J}_{q p r r}$.

The four matrices $\boldsymbol{J}_{q \text { rrr }}, \boldsymbol{J}_{x \text { rrr }}, \boldsymbol{J}_{q a c t}$ and $\boldsymbol{J}_{q \text { prr }}$ are defined as follows:

$$
\begin{gathered}
\boldsymbol{J}_{q r r r}=\left(\begin{array}{ccc}
\boldsymbol{v}_{1} \bullet \boldsymbol{u}_{\text {ortho } 1} & 0 & 0 \\
0 & \boldsymbol{v}_{2} \bullet \boldsymbol{u}_{\text {ortho } 2} & 0 \\
0 & 0 & \boldsymbol{v}_{3} \bullet \boldsymbol{u}_{\text {ortho } 3}
\end{array}\right), \\
\boldsymbol{J}_{\boldsymbol{x} r \mathrm{rr}}=\left(\begin{array}{ccc}
v_{x 1} & v_{y 1} & \left(\boldsymbol{C}_{1} \boldsymbol{D} \times \boldsymbol{v}_{1}\right)_{z} \\
v_{x 2} & v_{y 2} & \left(\boldsymbol{C}_{2} \boldsymbol{D} \times \boldsymbol{v}_{2}\right)_{z} \\
v_{x 3} & v_{y 3} & \left(\boldsymbol{C}_{3} \boldsymbol{D} \times \boldsymbol{v}_{3}\right)_{z}
\end{array}\right), \\
\boldsymbol{J}_{q \text { act }}=\left(\begin{array}{ccc}
\left(\boldsymbol{w}_{1} \bullet \boldsymbol{s}_{1}\right) & 0 & 0 \\
0 & \left(\boldsymbol{w}_{2} \bullet \boldsymbol{s}_{2}\right) & 0 \\
0 & 0 & \left(\boldsymbol{w}_{3} \bullet \boldsymbol{s}_{3}\right)
\end{array}\right), \\
\boldsymbol{J}_{q \text { prr }}=\left(\begin{array}{ccc}
J_{q \text { prr } 11} & 0 & 0 \\
0 & J_{q \text { prr } 22} & 0 \\
0 & 0 & J_{q \text { prr 33 }}
\end{array}\right)
\end{gathered}
$$

and

where

$$
\begin{gathered}
J_{q \text { prr } 11}=\left(\left(L_{a c t} \boldsymbol{u}_{\text {ortho1 }}-L_{4} \boldsymbol{u}_{1}\right) \bullet \boldsymbol{s}_{1}\right), \\
J_{q \text { prr } 22}\left(\left(L_{\text {act }} \boldsymbol{u}_{\text {ortho2 }}-L_{4} \boldsymbol{u}_{2}\right) \bullet \boldsymbol{s}_{\mathbf{2}}\right),
\end{gathered}
$$

and

$$
J_{\text {qact } 33}\left(\left(L_{\text {act }} \boldsymbol{u}_{\text {ortho3 }}-L_{4} \boldsymbol{u}_{3}\right) \bullet \boldsymbol{s}_{3}\right) \text {. }
$$

Note: - represents the dot product, and $\times$ represents the cross product.

The obtained matrix $\boldsymbol{J}_{\text {micro }}$ will be used later in III.B. 


\section{FLEXIBLE LINK MODELING AND EVALUATION OF FORCES}

In this part, we will introduce the flexible circular notch hinge, detail its characteristics, and present a modeling of the efforts generated by such a kind of link for the microstage.

\section{A. Flexible circular notch hinge}

\section{1) Theoretical aspect}

After a study of the different possibilities to realize flexible joints ([3] and [4]), we decided to model all the flexible joints as revolute joints $\mathrm{R}$, and to realize these flexible links as flexible circular notch hinge (see Fig. 4). Indeed, these joints are easy to study and to manufacture (much less parts than prismatic flexible joints), and they are well adapted to multiple instances of flexible links.

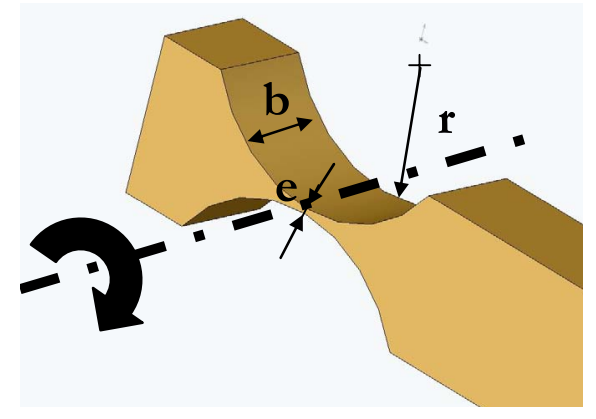

Fig. 4 Modeling of a flexible circular notch hinge

The two main characteristics of such a link stand in its angular stiffness $K_{\alpha}$ and its angular stroke $\alpha$ (defined in (3)), both depending on the chosen material and on the geometrical properties of the link: $e, b, r$.

$$
K_{\alpha}=\frac{2 E b e^{2,5}}{9 \pi \sqrt{r}} \text { and } \alpha=\frac{3 \pi \sigma_{a d m} \sqrt{r}}{4 E \sqrt{e}}
$$

The dimensions chosen for the flexible links are $e=0.5 \mathrm{~mm}, r=30 \mathrm{~mm}$ and $b=10 \mathrm{~mm}$ (these dimensions are consistent with the recommendations for standard flexible circular notch hinge described by Henein [4]). The chosen material is Alu7075 (high elastic limit aluminum alloy), with $E=72,000 \mathrm{MPa}$ and $\sigma_{a d m}=500 \mathrm{MPa}$. Thus, we obtain the following characteristics for the flexible links:

$$
K_{\alpha}=1,64 \mathrm{~N} \cdot \mathrm{m} / \mathrm{rad} \text { et } \alpha=7,26^{\circ}
$$

The value of $\alpha$ allows us to verify and validate the choice of this type of flexible link and also the choice of the material for the machining of the flexible parts. Indeed, it is very important to verify that the angular strokes of the revolute joints $\mathrm{R}$ for the whole micro-stage we found thanks to the inverse position kinematics solutions are smaller than $\alpha$. This is correct because the maximum angular stroke occurs at point $\mathrm{B}$ with angle $p$ : it is $4^{\circ}$. The angular stiffness $K_{\alpha}$ will be used in the modeling and evaluation of forces generated by the flexible links.

It is also possible to validate these results through an FEM analysis, but one has to be careful because of the big thickness change in the considered parts.

\section{B. Modeling of the forces of the micro-stage}

The micro-stage forces come from two different sources. Some come from the external forces applied to the traveling plate, and some result from the flexible links inside the mechanism. To be able to make a choice for the linear actuator, we need to know the amount of forces the actuator will have to push. So both kind of forces need to be investigated and expressed at the actuator.

\section{1) External forces}

External forces exerted on the traveling plate can be mapped onto actuator forces thanks to the force/velocity duality property:

$$
\boldsymbol{F}_{\text {act ext }}=\boldsymbol{J}_{\text {micro }}{ }^{T} \boldsymbol{F}_{\text {ext }}
$$

$\boldsymbol{J}_{\text {micro }}$ has been defined in (2), its dimension is $3 \times 3$. $\boldsymbol{F}_{\text {act ext }}$ represents the vector of forces generated by the external forces and expressed at the actuator origin $\mathrm{G}$, its dimension is $3 \times 1 . \boldsymbol{F}_{\text {ext }}$ represents the vector of external forces applied on the micro-stage and expressed at the center of the traveling plate $\mathrm{D}$, its dimension is $3 \times 1$. These two vectors are defined as follows:

$$
\boldsymbol{F}_{\text {act ext }}=\left(\begin{array}{l}
F_{\text {act ext } 1} \\
F_{\text {act ext } 2} \\
F_{\text {act ext } 3}
\end{array}\right) \text { and } \boldsymbol{F}_{\text {ext }}=\left(\begin{array}{c}
m a_{x} \\
m a_{y} \\
J_{z} \gamma_{z}
\end{array}\right)
$$

Where $m$ represents the mass of the macro-stage, $a_{x}$ and $a_{y}$ relatively represents the $\boldsymbol{x}$ and $\boldsymbol{y}$ components of the linear acceleration vector $\boldsymbol{a} \cdot \gamma_{z}$ represents the angular acceleration and $J_{z}$ represents the inertia moment around the $\boldsymbol{z}$ axis.

\section{2) Forces due to flexible links}

In the modeling of forces, flexible circular notch hinge can be considered as torsional springs, and then the efforts in flexible links can be modeled as a pure torque:

$$
\Delta C_{f l e x}=K \Delta \theta
$$

where $\Delta \boldsymbol{C}_{\text {flex }}$ represents the vector of torque generated at each flexible link, its dimension is $15 \times 1$. $\boldsymbol{K}$ represents the stiffness matrix composed of the stiffness of each flexible link on its diagonal elements, its dimension is $15 \times 15$. And $\boldsymbol{\Delta \theta}$ represents the vector of angular variation at each flexible link, its dimension is $15 \times 1$.

It is also possible to express the angular variation at each link as a function of the variation of position of the point of interest of the traveling plate D thanks to $\boldsymbol{M}^{-1}$ :

$$
\Delta \theta=M^{-1} \Delta x
$$

where $\Delta \boldsymbol{x}$ is the vector representing the variation of position of point $\mathrm{D}$, its dimension is $3 \times 1$ and it is defined as follow:

$$
\boldsymbol{\Delta x}=\left(\begin{array}{c}
\Delta x \\
\Delta y \\
\Delta \theta
\end{array}\right)
$$


The matrix $\boldsymbol{M}^{-1}$ will be defined later in III.B.3), its dimension is $15 \times 3$. Thanks to the force/velocity duality property, (7) is equivalent to:

$$
\Delta F_{f l e x}=\left(M^{-1}\right)^{T} \Delta C_{f l e x}
$$

where $\Delta \boldsymbol{F}_{\text {flex }}$ is the vector representing the efforts generated by the flexible links, and expressed at the centre of the traveling plate $\mathrm{D}$, its dimension is $3 \times 1 . \Delta \boldsymbol{F}_{\text {flex }}$ can be expressed as follows:

$$
\Delta \boldsymbol{F}_{\text {flex }}=\left(\begin{array}{l}
\Delta F_{\text {flex } x} \\
\Delta F_{\text {flex } y} \\
\Delta C_{\text {flex } z}
\end{array}\right)
$$

Combining (6), (7) and (8), we finally obtain an evaluation of the forces due to flexible links, and expressed at point $\mathrm{D}$.

$$
\Delta F_{\text {flex }}=\left(M^{-1}\right)^{T} K M^{-1} \Delta x
$$

Then, is it possible to express these forces at the actuator origin $\mathrm{G}$ using the following relation:

$$
F_{\text {act flex }}=\boldsymbol{J}_{\text {micro }}{ }^{\boldsymbol{T}} \boldsymbol{F}_{\text {flex }}
$$

where $\boldsymbol{F}_{\text {act flex }}$ represents the vector of forces generated by the flexible links and expressed at the actuator origin $G$, its dimension is $3 \times 1 . \boldsymbol{F}_{\text {act flex }}$ is defined as follows:

$$
\boldsymbol{F}_{\text {act flex }}=\left(\begin{array}{l}
F_{a c t \text { flex } 1} \\
F_{a c t \text { flex } 2} \\
F_{\text {act flex } 3}
\end{array}\right)
$$

\section{3) Expression of the matrix $\boldsymbol{M}^{-1}$}

To express the matrix $\boldsymbol{M}^{-1}$, we will separate the microstage into two sub-mechanisms, as we did for the models of the inverse positions and velocities (see [2]). One is composed of the planar parallel 3-RRR mechanism represented in Fig. 1 by the letters $\mathrm{A}_{\mathrm{i}} \mathrm{B}_{\mathrm{i}} \mathrm{C}_{\mathrm{i}} \mathrm{D}_{\mathrm{i}}(i=1,2,3$ stands for the number of the kinematic chain), and the second one is the decoupled actuating PRR mechanism represented in Fig. 1 by the letters $\mathrm{A}_{\mathrm{i}} \mathrm{E}_{\mathrm{i}} \mathrm{F}_{\mathrm{i}} \mathrm{G}_{\mathrm{i}}$.

a) Planar parallel 3-RRR mechanism $A_{i} B_{i} C_{i} D_{i}$

With this sub-mechanism, the velocity at point $\mathrm{C}_{\mathrm{i}}$ can be expressed with two different ways. We can express the velocities at points $A_{i}, B_{i}$, and $C_{i}$ as a function of the velocity of the point of interest of the traveling plate $\mathrm{D}$. We obtain the following relation for one kinematic chain $i$ :

with

$$
\begin{gathered}
\dot{\boldsymbol{q}}_{c h i}=\boldsymbol{J}_{c h i}{ }^{-1} \dot{\boldsymbol{x}} \\
\dot{\boldsymbol{q}}_{c h i}=\left(\begin{array}{l}
\dot{\theta}_{A i} \\
\dot{\theta}_{B i} \\
\dot{\theta}_{C i}
\end{array}\right) \text { and } \dot{\boldsymbol{x}}=\left(\begin{array}{c}
\dot{x} \\
\dot{y} \\
\dot{\theta}
\end{array}\right)
\end{gathered}
$$

and

$$
J_{c h i}^{-1}=\left(J_{q i}\right)^{-1} J_{x i}
$$

where $\dot{\boldsymbol{q}}_{c h i}$ represents the vector of velocity of one chain of the planar parallel 3-RRR mechanism, that is to say the velocities at points $A_{i}, B_{i}$ and $C_{i}$, its dimension is $3 \times 1$. The matrices $\boldsymbol{J}_{\boldsymbol{q} i}$ and $\boldsymbol{J}_{\boldsymbol{x} i}$ are defined as follows:

$$
\begin{gathered}
\boldsymbol{J}_{\boldsymbol{q} i}=\left(\begin{array}{ccc}
\left(\left(l_{1} \boldsymbol{u}_{\text {orthoi }}+l_{2} \boldsymbol{v}_{\text {orthoi }}\right) \cdot \boldsymbol{x}\right) & \left(l_{2} \boldsymbol{v}_{\text {ortho }} \cdot \boldsymbol{x}\right) & 0 \\
\left(\left(l_{1} \boldsymbol{u}_{\text {orthoi }}+l_{2} \boldsymbol{v}_{\text {orthoi }}\right) \cdot \boldsymbol{y}\right) & \left(l_{2} \boldsymbol{v}_{\text {ortho }} \cdot \boldsymbol{y}\right) & 0 \\
1 & 1 & 1
\end{array}\right) \\
\boldsymbol{J}_{\boldsymbol{x} i}=\left(\begin{array}{ccc}
1 & 0 & \left(\boldsymbol{E}_{i} \boldsymbol{C}_{i} \cdot \boldsymbol{y}\right) \\
0 & 1 & -\left(\boldsymbol{E}_{i} \boldsymbol{C}_{i} \cdot x\right) \\
0 & 0 & 0
\end{array}\right)
\end{gathered}
$$

and

Note that the dimension of $\boldsymbol{J}_{c h i}{ }^{-1}$ is $3 \times 3$.

b) Decoupled actuating $\underline{P R R}$ mechanism $A_{i} E_{i} F_{i} G_{i}$

Writing the velocity at point $\mathrm{E}_{\mathrm{i}}$ with two different ways, we can obtain the following relations:

$$
\left(\begin{array}{c}
\dot{\theta}_{E i} \\
\dot{\theta}_{F i}
\end{array}\right)=\boldsymbol{J}_{\text {rodi }} \dot{\theta}_{A i}
$$

and

$$
\boldsymbol{J}_{\text {rodi }}=\boldsymbol{J}_{a i}{ }^{-1} \boldsymbol{J}_{b i}
$$

where the matrices $\boldsymbol{J}_{\boldsymbol{a} i}$ and $\boldsymbol{J}_{\boldsymbol{b} i}$ are defined as follows:

$$
\begin{aligned}
\boldsymbol{J}_{a i} & =\left(\begin{array}{cc}
1 & 1 \\
0 & \left(\left(\boldsymbol{E}_{i} \boldsymbol{F}_{i} \times \boldsymbol{z}\right) \bullet \boldsymbol{w}_{\text {ortho }}\right)
\end{array}\right) \\
\text { and } & \boldsymbol{J}_{\boldsymbol{b i}}=\left(\begin{array}{c}
\left(l_{1} \boldsymbol{u}_{\text {iortho }}-l_{4} \boldsymbol{u}_{i}\right) \bullet \boldsymbol{w}_{\text {ortho }} \\
1
\end{array}\right)
\end{aligned}
$$

With (11), we know how to express the velocity at point $A_{i}$ in function of the velocities of the point of interest of the traveling plate D. Indeed, if we call $\boldsymbol{L}_{j c h i}$ ( $j=1,2,3)$ the horizontal vector representing the $j^{\text {th }}$ line of matrix $\boldsymbol{J}_{\boldsymbol{c h i}}$, we obtain the following relation:

$$
\dot{\theta}_{A i}=\boldsymbol{L}_{1 \text { chi }} \dot{\boldsymbol{x}}
$$

Hence, we can merge (13) and (15) to obtain:

$$
\left(\begin{array}{l}
\dot{\theta}_{E i} \\
\dot{\theta}_{F i}
\end{array}\right)=\boldsymbol{J}_{\text {rodi }} \boldsymbol{L}_{\boldsymbol{1 ~ c h i}} \dot{\boldsymbol{x}}
$$

c) Complete micro-stage mechanism

We can merge the results found in (11) and (16). We obtain the following relation:

$$
\left(\begin{array}{l}
\dot{\theta}_{A i} \\
\dot{\theta}_{B i} \\
\dot{\theta}_{C i} \\
\dot{\theta}_{E i} \\
\dot{\theta}_{F i}
\end{array}\right)=\left(\begin{array}{c}
\boldsymbol{J}_{\text {ch } i}^{-1} \\
\boldsymbol{J}_{\text {rodi }} \boldsymbol{L}_{\boldsymbol{l} \text { chi }}
\end{array}\right)\left(\begin{array}{c}
\dot{x} \\
\dot{y} \\
\dot{\theta}
\end{array}\right)
$$

This gives us the expression of matrix $\boldsymbol{M}_{\boldsymbol{i}}^{-1}$ for one kinematic chain $i$, which is:

$$
M_{i}^{-1}=\left(\begin{array}{c}
J_{c h i}^{-1} \\
J_{r o d i} L_{1 c h i}
\end{array}\right)
$$

Finally, for the complete micro-stage (3 kinematic chains), we obtain the following matrix $\boldsymbol{M}^{-1}$ : 


$$
M^{-1}=\left(\begin{array}{c}
J_{c h 1}{ }^{-1} \\
J_{r o d l} L_{l c h i} \\
J_{c h 2}-1 \\
J_{r o d 2} L_{1 c h i} \\
J_{c h 2} 2^{-1} \\
J_{r o d 3} L_{1 c h i}
\end{array}\right)
$$

The obtaining of matrices $\boldsymbol{J}_{\boldsymbol{c h i}}{ }^{-1}$ et $\boldsymbol{J}_{\text {rodi }}$ is detailed in (12) and (14).

\section{4) Total forces of the micro-stage}

By superposition of (5) and (10), we can obtain the total force at the actuators origin:

$$
\boldsymbol{F}_{\text {act total }}=\boldsymbol{F}_{\text {actext }}+\boldsymbol{F}_{\text {act flex }}
$$

\section{Simulations and results}

We run some simulations using Matlab ${ }^{\circledR}$. In these simulations, the point of interest of the traveling plate $D$ covers the complete workspace, that is to say a disc of diameter $\varnothing 0.2 \mathrm{~mm}$ with an orientation of $\pm 0.1^{\circ}$. Then, we have been able to evaluate the forces in the micro-stage.

The moving mass of the macro-stage is about $50 \mathrm{~kg}$, and its linear acceleration is $0.5 \mathrm{~m} . \mathrm{s}^{-2}$. The angular acceleration is insignificant and will be considered insignificant. The maximum force obtained after simulations is $F_{\text {act ext }}=4.04 \mathrm{~N}$.

With the stiffness we calculated before (we took the same angular stiffness value $K_{\alpha}=1,64 \mathrm{~N} . \mathrm{m} / \mathrm{rad}$ for each flexible link of the micro-stage), the maximum force generated by the flexible links is $F_{\text {act flex }}=0.0034$ N. It is interesting to note that the actuator force due to flexible links represent about $0.1 \%$ of the actuator force due to external force. These forces can be neglected in the control of the machine compared to the external ones.

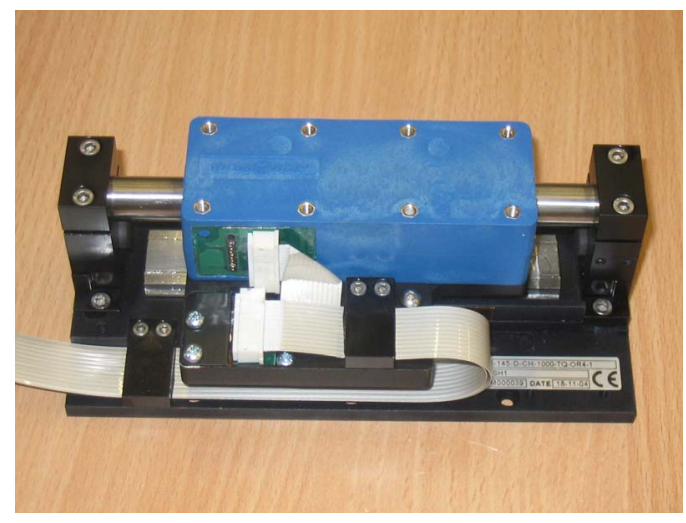

Fig. 5 Chosen micro-actuator

The total actuator (see (18)) is about $F_{\text {act total }}=4.05 \mathrm{~N}$. The actuators have been selected thanks to the time cycle we expect (less than $0.5 \mathrm{~s}$ ) and the forces that are required to be generated.

We choose some micro-actuators that have a stroke of 2 $\mathrm{mm}$ and that can push up to $7 \mathrm{~N}$ (see Fig. 5). The actuators encoders have a resolution of $0.1 \mu \mathrm{m}$, and thanks to the geometry we used, we expect to be able to generate a resolution of displacement of about $16 \mathrm{~nm}$ (reduction ratio of 6.2, see Part I).

\section{IV.DESIGN AND MANUFACTURING OF THE MICRO-STAGE}

\section{A. Presentation}

The micro-stage has been designed with SolidWorks 2004® and then manufactured and assembled. Fig. 6 represents a top $\mathrm{CAD}$ view of the micro-stage. We can see on this CAD view the representation of one kinematic chain of the micro-stage as it has been presented in Fig. 1.

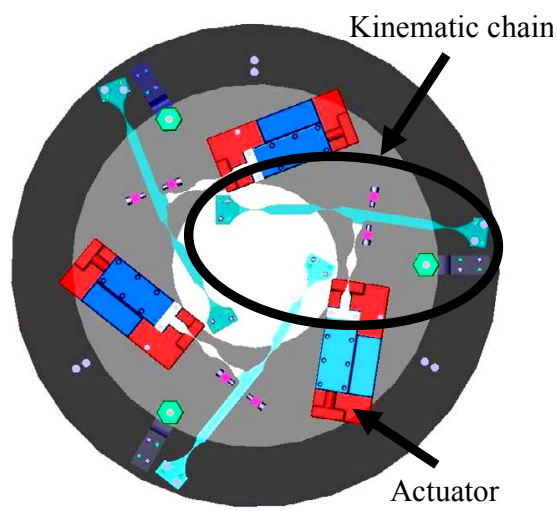

Fig. 6 Top CAD view of the micro-stage

Fig. 7 represents another 3D CAD view of the mechanism. In this picture, we can observe that some mechanical limit stops have been integrated to the final design of the micro-stage. The function of these mechanical limit stops is to avoid any possible deterioration of the flexible links due to a too large displacement.

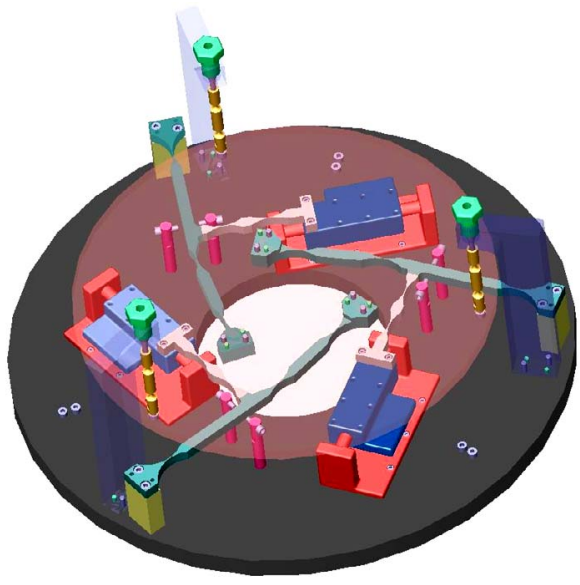

Fig. 7 3D CAD view of the micro-stage

Fig. 8. represents the complete micro-stage mounted on the macro-stage. This gives us a real view of the complete high resolution and fast positioning mechanism.

\section{B. Particularity of the support system}

The support system is designed to support the traveling plate in three points, so that the flexible links (RRR and PRR) only works in a rotational motion around an axis normal to the in plane motion. The support of the traveling 
plate is ensured by a spherical-spherical link (see Fig. 9) so that in fact the traveling plate moves according to a translation and a rotation. The translation is circular and the rotation helicoidal. But as the workspace of the traveling space is very small (disc of diameter $\varnothing 0,2 \mathrm{~mm}$ with an orientation of $\pm 0,1^{\circ}$ ), the vertical translation generated by these two motions should remain insignificant: $125 \mathrm{~nm}$.

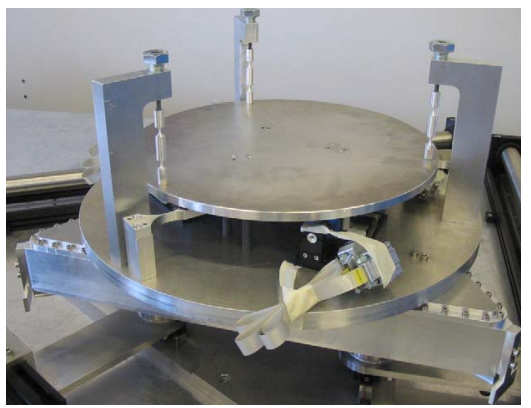

Fig. 8 Complete micro-stage with support in three points

The spherical-spherical link has been designed by removing material on a spherical shape to let a thickness of $2 \mathrm{~mm}$. The forces necessary to move this support mechanism that is also flexible have been estimated using finite element analysis (another possibility of modeling would have been to use the method we described in this article) and found equal to about $0.1 \mathrm{~N}$ per support. This results into a force of $0.3 \mathrm{~N}$ for the complete support system, which is equivalent to a force of $0.016 \mathrm{~N}$ by actuator. One more time, theses forces are insignificant compared to the one generated by the external forces (mainly inertia).

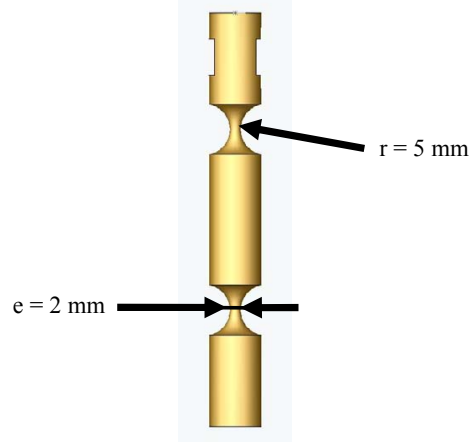

Fig. 9 Support system of the traveling plate

\section{CONCLUSION}

A novel high-resolution and high-speed precision positioning mechanism has been presented, based on a planar parallel quasi-singular flexible architecture, with a decoupled actuating mechanism thanks to a linear actuator. The presentations of the architecture and geometry have been detailed by the authors in Part I [2]. The models for inverse position and velocity solutions have been derived.

In this paper, we have addressed the important issue of the efforts modeling for the micro-stage. Two kinds of efforts have been taken into account. The ones generated by the flexible links (flexible circular notch hinge) and those generated by the external efforts applied on the micro-stage. These efforts have been modeled and evaluated through simulations for the micro-stage. Hence, it has been possible to make a choice for the actuator and also to design, manufacture and assemble the complete micro-stage. The resolution of positioning for this microstage is expected to be around $16 \mathrm{~nm}$ with the use of integrated actuator scales which possess a resolution of 0.1 $\mu \mathrm{m}$.

\section{FUTURE WORK}

The control of this micro-stage mechanism, as well as the control of the complete machine (macro-stage + microstage) will be realized soon. We use the package composed of the RTX software (Real Time eXtension) running under Windows, and a PC I/O board designed and realized at the LIRMM. We will realize the control of both stages independently first, then we will synchronize the two stages. The, experiments will be conducted to measure the real resolution of the system. Finally, we will add an external measuring system (local interferometry measuring systems www.optra.com) to obtain a better positioning resolution by measuring the actual real position of the point of interest without using the actuators' scales. Indeed, the overall accuracy will be guaranteed by this external measuring system.

\section{REFERENCES}

[1] Ronchi S., Company O., Pierrot F., and Fournier A., "PRP Planar Parallel Mechanism in Configurations Improving Displacement Resolution", in proceedings of the 1st International Conference on Positioning Technologies, Hamamatsu, Japan, pp.279-284, June 09-11 (2004).

[2] Ronchi S., Company O., Krut S., Pierrot F., and Fournier A., "High Resolution Flexible 3-RRR Planar Parallel Micro-Stage in Near Singular Configuration for Resolution Improvement (Part I)", submitted to IROS 2005, Edmonton, Canada, 6p., August 2-6 (2005).

[3] Howell L. L., Compliant Mechanisms, John Wiley \& Sons Inc, New York, 459p. (2001).

[4] Henein S., Conception des Guidages Flexibles, Presse Polytechniques et Universitaires Romandes, Schüler SA, Lausanne, 225p. (2004).

[5] Chevalier L., and Konieczska S., "Liaisons Elastiques : Calculs et Applications", ENS Cachan, 21p.

[6] Harai T., Herve J. M., and Tanikawa T., "Development of 3 DOF Micro Finger", in Proceedings of IROS : Intelligent Robots and Systems 96, Osaka, Japan, Vol. 2, pp.981-987, November 5-8 (1996).

[7] Chung G. B., Yi B.-J., Suh I. K., Kim W. K., and Chung W. K., "Design and Analysis of a Spatial 3-DOF Micromanipulator for TeleOperation", in Proceedings of the 2001 IEEE/RSJ International Conference on Intelligent Robots and Systems, Maui, Hawaii, USA, pp.337-342, October 29 - November 03 (2001).

[8] Hesselbach J., Raatz A., and Kunzmann H., "Perdormance of PseudoElastic Flexure Hinges in Parallel Robots for Micro-Assembly Tasks", in Proceedings of the CIRP, pp.329-332, (2004).

[9] Yi B.-J., Chung G. B., Na H. Y., Kim W. H., and Suh I. H., "Design and Experiment of a 3-DOF Parallel Micromechanism Utilizing Flexure hinges", in Proceedings of IEEE Transactions on Robotics and Automation, Las Vegas, Nevada, pp.604-612, August (2003).

[10] Ranganath R., Nair P. S., Mruthyunjaya T. S., and Ghosal A., "A Force-Torque Sensor Based on a Stewart Platform in a Near Singular Configuration", Journal of Mechanism and Machine theory, Vol. 39, pp.971-998, April 10 (2004). 